Erratum

\title{
Erratum to "The Role of KRAS Mutational Analysis to Determine the Site of Origin of Metastatic Carcinoma to the Lung: A Case Report"
}

\author{
Ahmad Alkhasawneh, ${ }^{1}$ Hui-Jia Dong, ${ }^{1}$ Chen Liu, ${ }^{1}$ Carmen Allegra, ${ }^{2}$ and Robert W. Allan ${ }^{1}$ \\ ${ }^{1}$ Department of Pathology, Immunology, and Laboratory Medicine, College of Medicine, University of Florida, P.O. Box 100275, \\ 1600 SW Archer Road, Gainesville, FL 32610, USA \\ ${ }^{2}$ Division of Hematology and Oncology, College of Medicine, University of Florida, 1600 SW Archer Road, Gainesville, FL 32610, USA \\ Correspondence should be addressed to Ahmad Alkhasawneh; ahm0001846@ufl.edu
}

Received 5 April 2013; Accepted 14 April 2013

Copyright (C) 2013 Ahmad Alkhasawneh et al. This is an open access article distributed under the Creative Commons Attribution License, which permits unrestricted use, distribution, and reproduction in any medium, provided the original work is properly cited.

In the paper titled "The Role of KRAS Mutational Analysis to Determine the Site of Origin of Metastatic Carcinoma to the Lung: A Case Report," the author's name Ahmad Alkhasawneh was incorrectly listed as Ahmed N. Alkhasawneh; here it is corrected. 


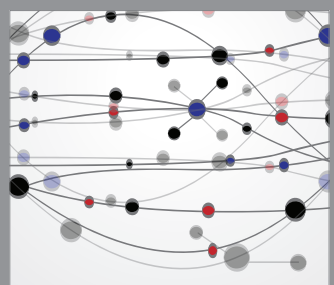

The Scientific World Journal
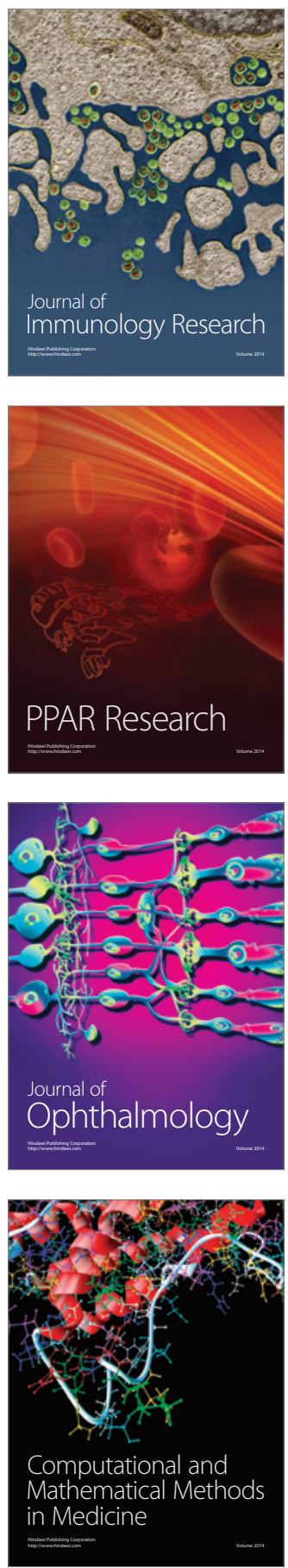

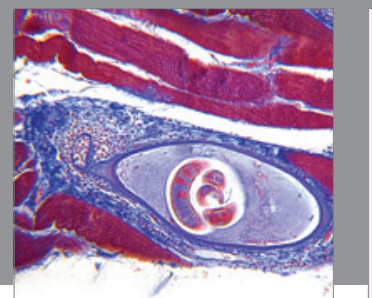

Gastroenterology

Research and Practice
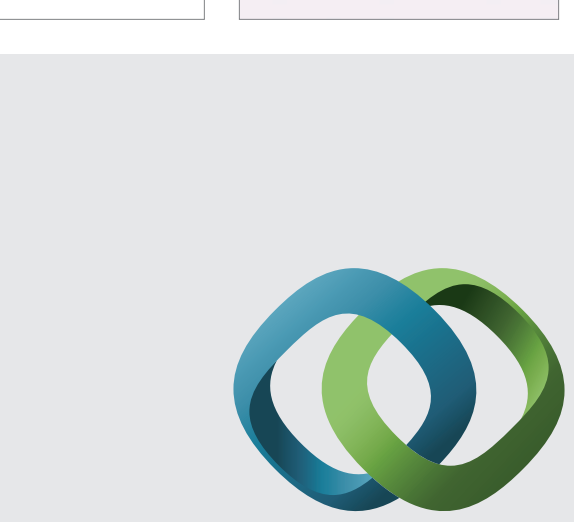

\section{Hindawi}

Submit your manuscripts at

http://www.hindawi.com
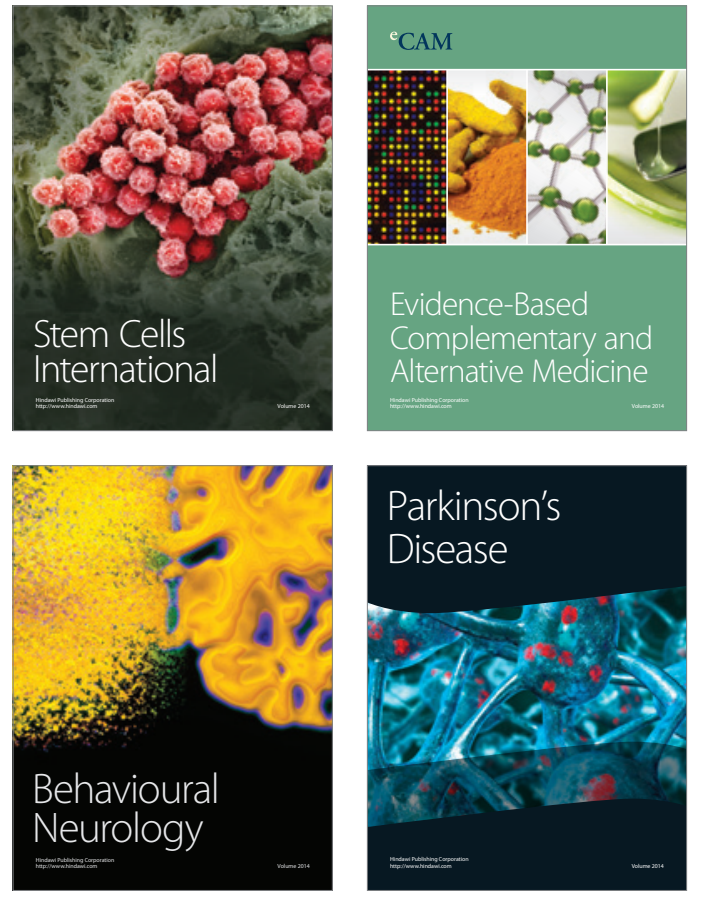
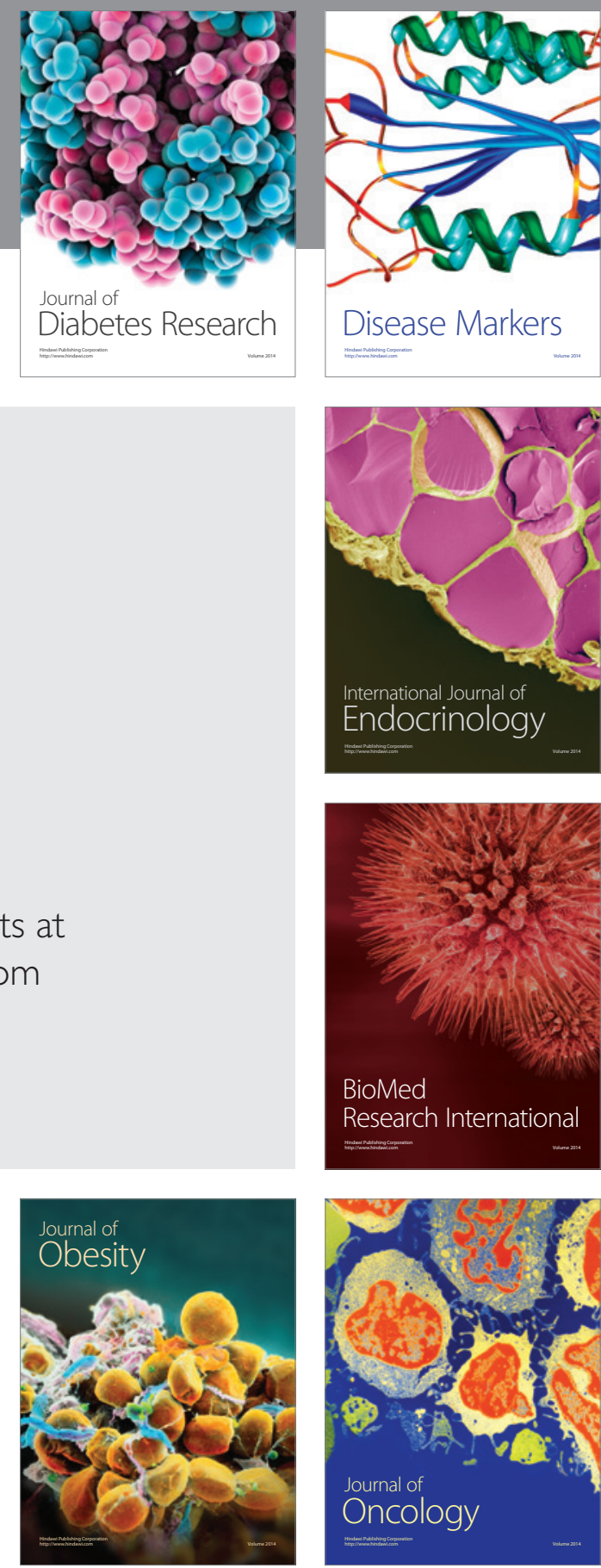

Disease Markers
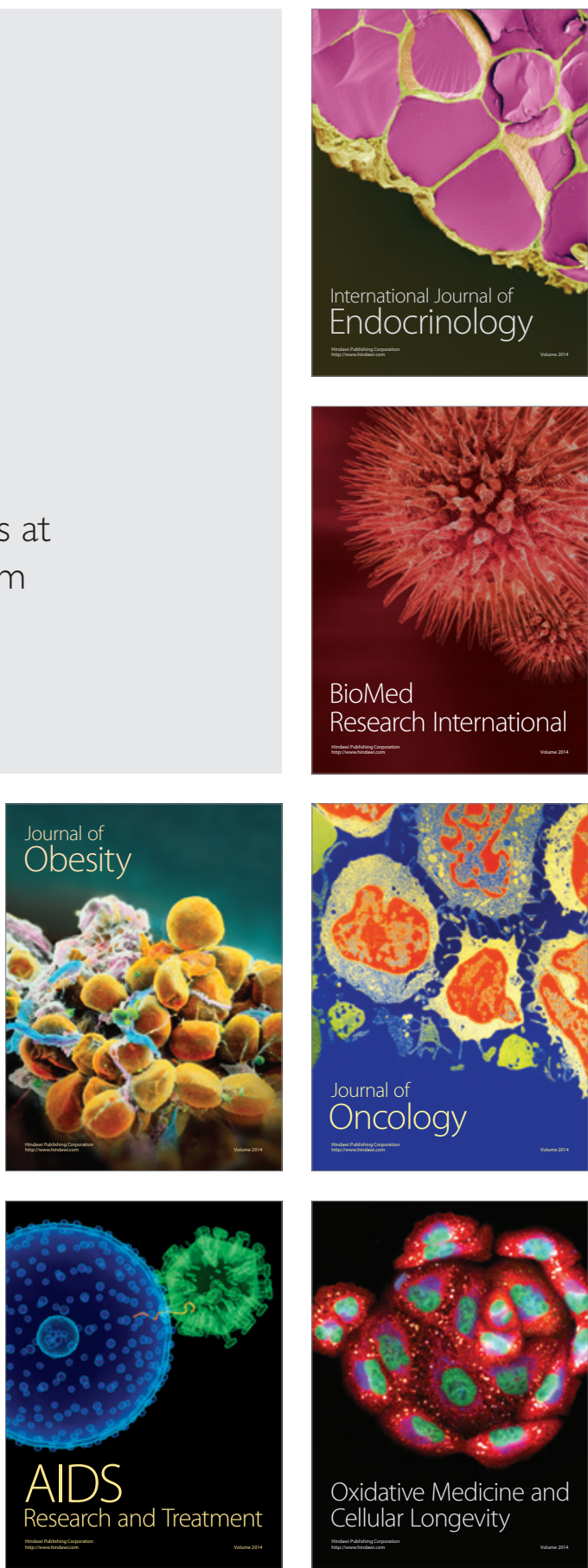